\title{
Addressing a system failure to diagnose COPD and Asthma
}

C Michael Roberts, Queen Mary University of London, UK

James Calvert, Aneurin Bevan University Health Board, UK

Katherine Hickman, GP \& Primary Care Lead for NACAP, Respiratory Lead West Yorkshire \& Harrogate ICS

Ian P Sinha, University of Liverpool, UK

Sally J Singh Department of Respiratory Science, University of Leicester, UK

C Michael Roberts, Queen Mary University of London, UK

John R Hurst, UCL Respiratory, University College London, London, UK

All authors are also affiliated to the Royal College of Physicians of London UK

In order to provide high quality guideline based care for asthma or COPD we must first establish the correct diagnosis. The National Asthma and COPD Audit Programme (NACAP) has highlighted a significant issue across England, Scotland and Wales that potentially undermines care for many people with airways disease.

The clinical diagnosis of COPD requires confirmation with quality assured spirometry performed following administration of a bronchodilator. It should demonstrate airflow obstruction defined as a ratio of the forced expiratory volume in 1 second (FEV1) over the forced vital capacity (FVC) of < 0.7. 1,2 [NICE QS \& Guide 2016] The national audit demonstrates that for the $>275,000$ episodes of acute COPD admissions entered, less than half have a record of spirometry, from current or past hospital records, available to the team treating the exacerbation of COPD. In 14\% of cases, where a spirometry result is available, that result is inconsistent with the diagnosis, but the patient is still treated for and discharged with a COPD label. ${ }^{3}$ [COPD clinical report 2020] At 90 days post discharge $12 \%$ of audited patients have died, half from a non-COPD cause, and $47 \%$ readmitted, $53 \%$ with a non-COPD diagnosis and many still without a record of spirometry. ${ }^{4}$ [COPD outcomes report 2020]. Pulmonary rehabilitation for COPD patients, is an evidence based intervention heavily promoted in the NHS Long Term Plan. Only $50 \%$ of the 12,000 cases of enrolled 'COPD' patients in the most recent reporting have a record of spirometry to confirm the diagnosis at referral. ${ }^{5}$ [PR clinical report 2020]

For asthma acute admissions the issue is even more complex. For children under the age of 5 there is no guideline specification for a diagnostic test of asthma. Within NACAP 15,000 cases were entered into the audit more than double those recorded in the corresponding hospital episode statistics where coding had defined such cases as wheeze or viral bronchitis. For older children and young people (CYP) the situation is not much better with less than 1 in 5 having any peak expiratory flow (PEF) measurement made during the course of their admission. ${ }^{6}$ [CYP clinical report 2021] In adult asthma there is also disagreement regarding diagnostic criteria which should be confirmed by empiric testing according to NICE guidance but which is fundamentally a clinical diagnosis within BTS/SIGN guidelines. Both recommend PEF testing to assess severity of an attack at hospital admission and to guide management. In the sample of 37,000 cases from 173 hospitals 
entered in NACAP only $28 \%$ had a PEF recorded as part of an initial assessment (in the first hour) and $15 \%$ had no PEF recorded at any time during their admission. ${ }^{7}$ [AA report clinical]

It may be argued that the lack of secondary care diagnostic data for these patients is because primary care now holds the diagnosis for airflow obstruction. The 2020 Welsh primary care audit of 226 practices including nearly 50,000 'diagnosed' asthma and COPD patients challenges this assumption. Of COPD patients diagnosed within the last 2 years, only $44 \%$ had a code recorded for a spirometry test and only $11.5 \%$ the gold standard post bronchodilator test with an obstructed ratio. [Primary Care 2020] The 2018 Welsh primary care audit showed that around $25 \%$ of spirometric ratios coded as 'confirming a diagnosis of COPD' had a ratio incompatible with that diagnosis. [Fisk 2018] For those on asthma registers the picture is a little better with $76 \%$ newly diagnosed adult patients having a confirmatory test of some kind recorded, PEF, Spirometry, or exhaled Nitric Oxide, whilst amongst those aged 6-18 years this falls to 67\%. [Primary care 2020] The clinical accuracy of a diagnosis of COPD compared to one confirmed by spirometry is known to lie between 60 and $75 \%$. [Jones 2008 Stoloff 2011 Casas 2016 Wu 2017] We would not accept that under half of acute admissions with myocardial infarction were treated on clinical suspicion without diagnostic testing, or that a fifth of patients with diabetes be managed without reference to an HBA1C level. We would be dismayed if half of cardiac and diabetic patients were managed in primary care as long term conditions without any confirmatory laboratory diagnostic testing, yet we do for respiratory patients.

The reasons for poor diagnosis of airways obstruction are multiple and complex ranging from lack of access to testing to diagnostic confusion amongst clinicians and poor implementation of guideline evidence. Rather than rehearse these in detail we suggest four practical improvement recommendations to colleagues:

1. Lead clinicians in each Trust should have responsibility for ensuring diagnostic accuracy is maintained. Working in collaboration with clinical coders, diagnosis is correctly entered into the discharge summary shared with community and primary care teams and with the patient and their carers. Collecting data via NACAP provides an obvious source to measure adherence.

2. Diagnostic data should be made available across health care interfaces through electronic records and should also be actively sought and recorded in clinical notes across primary secondary and community records. If necessary phoning up a GP surgery or physiology laboratory for a result.

3. Primary care clinicians should use diagnostic templates and search tools for their electronic records to ensure that diagnoses have been confirmed by appropriate objective tests, and where these are not compatible with the presumptive diagnosis, an alternative one be sought.

4. For cases of wheeze, cough and breathlessness, particularly, but not only in CYP, a presumptive diagnosis of asthma should be made, but with an understanding that over time that may be withdrawn or confirmed by a combination of clinical course and objective testing.

This is a call to action across the secondary, community, and primary care clinical pathway where integrated care requires us all to take responsibility to establish the correct diagnosis at the earliest opportunity. 


\section{References:}

3. Hurst J, Legg M, Shanahan L, Imoedemhe E, Andrews R, Moussaif M, Adamson A, Stone P, Quint J, Roberts CM. National Asthma and Chronic Obstructive Pulmonary Disease Audit Programme (NACAP). COPD clinical audit 2018/19 (people with COPD exacerbations discharged from acute hospitals in England, Scotland and Wales between September 2018 and 2019). Clinical audit report. London: RCP, July 2020.

2. NICE Quality Standards, Chronic Obstructive Pulmonary Disease in adults [QS10] (2016), statement 1: People aged over 35 years who present with a risk factor and one or more symptoms of chronic obstructive pulmonary disease (COPD) have post-bronchodilator spirometry.

1. NICE Guideline [NG115], Chronic obstructive pulmonary disease in over 16s: diagnosis and management, 1.1.5: Measure post-bronchodilator spirometry to confirm the diagnosis of COPD3

4. Hurst J, Shanahan L, Imoedemhe E, Andrews R, Moussaif M, Adamson A, Quint J, Roberts CM. National Asthma and Chronic Obstructive Pulmonary Disease Audit Programme (NACAP). Outcomes of patients included in the 2017/18 COPD clinical audit (patients with COPD exacerbations discharged from acute hospitals in England and Wales between September 2017 and September 2018). Audit report. London: RCP, 2020.

5. Singh S, Latchem S, Andrews R, Garnavos N, Long N, Stone P, Adamson A, Quint J, Roberts CM. National Asthma and Chronic Obstructive Pulmonary Disease Audit Programme (NACAP). Pulmonary rehabilitation audit report 2019. Combined clinical and organisational audit of pulmonary rehabilitation services in England, Scotland and Wales. London: RCP, 2020.

6. Sinha I, Latchem S, Amusan L, Andrews R, Afful S, Imoedemhe E, Yassin N, Bunning T, Stone P, Adamson A, Quint J, Roberts CM. National Asthma and COPD Audit Programme: Children and young people asthma clinical and organisational audit 2019/20. Combined clinical and organisational audit of children and young people asthma services in England, Scotland and Wales. London: RCP, 2021.

7. Calvert J, Latchem S, Andrews R, Imoedemhe E, Robinson S, Yassin N, Bunning T, Adamson A, Stone P, Quint J, Roberts CM. National Asthma and Chronic Obstructive Pulmonary Disease Audit Programme (NACAP): Adult asthma clinical audit 2019/20 (Adults with asthma attacks discharged from hospitals in England, Scotland and Wales between 1 April 2019 and 31 March 2020). Clinical audit report. RCP: London, 2021.

Hickman K, Amusan L, Mortier K, Stone P, Quint J, Roberts CM. National Asthma and COPD Audit Programme (NACAP). Wales primary care clinical audit 2020. Clinical audit report. London: RCP, 2021. Copyright (C) Healthcare Quality Improvement Partnership 2021 ISBN: 978-1-86016-839- 
Casas Herrera A, Montes de Oca M, López Varela MV, et al. COPD underdiagnosis and misdiagnosis in a high-risk primary care population in four Latin American countries. A key to enhance disease diagnosis: the PUMA Study. PLoS ONE. 2016;11(4):e0152266.

Fisk M, McMillan V, Brown J, Holzhauer-Barrie J, Khan MS, Baxter N, Roberts CM. Inaccurate diagnosis of COPD: the Welsh National COPD Audit. Br J Gen Pract. 2019 Jan;69(678):e1-e7. doi: 10.3399/bjgp18X700385. Epub 2018 Dec

Huimin Wu ${ }^{1}$, Robert A Wise ${ }^{2}$, Ann E Medinger ${ }^{3}$ Do Patients Hospitalized With COPD Have Airflow Obstruction? 2017 Jun;151(6):1263-1271. doi: 10.1016/j.chest.2017.01.003. Epub 2017 Jan 13.

Jones RC, Dickson-Spillmann M, Mather MJ, Marks D, Shackell BS. Accuracy of diagnostic registers and management of chronic obstructive pulmonary disease: the Devon primary care audit. Respir Res. 2008 Aug 18;9(1):62. doi: 10.1186/1465-9921-9-62.PMID: 18710575

Stoloff SWJ Diagnosis and treatment of patients with chronic obstructive pulmonary disease in the primary care setting: focus on the role of spirometry and bronchodilator reversibility. Fam Pract. 2011 Apr;60(4 Suppl Diagnosis):S9-16.PMID: 21472146.

White J, Paton J, Niven R, Pinnock H. BTS-SIGN-and-NICE-Asthma-guidelines.pdf (bmi.com) 